OPEN ACCESS

Edited by:

Jiuquan Zhang,

Chongqing University, China

Reviewed by:

Jianghua Shao,

Second Hospital of Nanchang, China

Di Dong,

Institute of Automation (CAS), China

*Correspondence: Sheng Yan

shengyan@zju.edu.cn

Lingxiang Ruan

1190026@zju.edu.cn

${ }^{\dagger}$ These authors have contributed equally to this work and share first authorship

Specialty section:

This article was submitted to

Cancer Imaging and Image-directed Interventions,

a section of the journal

Frontiers in Oncology

Received: 26 November 2020

Accepted: 31 August 2021

Published: 22 September 2021

Citation:

Liu X, Liang $X$, Ruan L and Yan S (2021) A Clinical-Radiomics Nomogram for Preoperative Prediction of Lymph Node Metastasis in Gallbladder Cancer.

Front. Oncol. 11:633852. doi: 10.3389/fonc.2021.633852

\section{A Clinical-Radiomics Nomogram for Preoperative Prediction of Lymph Node Metastasis in Gallbladder Cancer}

\author{
Xingyu Liu ${ }^{1 \dagger}$, Xiaoyuan Liang ${ }^{1 \dagger}$, Lingxiang Ruan ${ }^{2 *}$ and Sheng Yan ${ }^{1 *}$ \\ ${ }^{1}$ Department of Hepatobiliary and Pancreatic Surgery, The Second Affiliated Hospital Zhejiang University School of Medicine, \\ Hangzhou, China, ${ }^{2}$ Department of Radiology, The First Affiliated Hospital Zhejiang University School of Medicine, Hangzhou, China
}

Objectives: The aim of the current study was to develop and validate a nomogram based on CT radiomics features and clinical variables for predicting lymph node metastasis (LNM) in gallbladder cancer (GBC).

Methods: A total of $353 \mathrm{GBC}$ patients from two hospitals were enrolled in this study. A Radscore was developed using least absolute shrinkage and selection operator (LASSO) logistic model based on the radiomics features extracted from the portal venous-phase computed tomography (CT). Four prediction models were constructed based on the training cohort and were validated using internal and external validation cohorts. The most effective model was then selected to build a nomogram.

Results: The clinical-radiomics nomogram, which comprised Radscore and three clinical variables, showed the best diagnostic efficiency in the training cohort ( $A \cup C=0.851$ ), internal validation cohort ( $\mathrm{AUC}=0.819$ ), and external validation cohort ( $\mathrm{AUC}=0.824$ ). Calibration curves showed good discrimination ability of the nomogram using the validation cohorts. Decision curve analysis (DCA) showed that the nomogram had a high clinical utility.

Conclusion: The findings showed that the clinical-radiomics nomogram based on radiomics features and clinical parameters is a promising tool for preoperative prediction of $L N$ status in patients with GBC.

Keywords: gallbladder cancer, radiomics, computed tomography, lymph node metastasis, nomogram

\section{INTRODUCTION}

Gallbladder cancer (GBC) is the most common malignant tumor of the biliary tract, accounting for $80 \%-95 \%$ of biliary tract cancer cases in the world and is ranked the sixth among gastrointestinal cancers (1). GBC is commonly detected in patients along with cholecystitis; however, early diagnosis is challenging owing to quiet symptoms and limited imaging methods. Poor diagnosis results in low median overall survival and low 5-year survival rate $(2,3)$. Although surgery is associated with poor prognosis, it is the primary approach for treatment of patients with GBC (4). However, 
only $20 \%-30 \%$ of patients diagnosed in the clinic can undergo radical resection and the postoperative recurrence rate reaches $50 \%-70 \%$ owing to late diagnosis (5).

Lymph node metastasis (LNM) is the most important factor in clinical staging of GBC. Patients with positive LNM are classified as stage IIIb, based on the 8th edition of the American Joint Committee on Cancer (AJCC) gallbladder cancer staging system (GBC). Stage IIIb indicates worse prognosis compared with prognosis of earlier stages (6-8). Radical cholecystectomy including expanded systemic lymph node (LN) dissection is recommended to improve surgical outcome $(5,9)$. However, not all patients can benefit from radical lymphadenectomy. Previous studies reported that extended radical resection should not be conducted in patients with negative LNM because it may cause serious postoperative complications $(10,11)$. Conversely, patients diagnosed with extensive LNM can choose neoadjuvant therapy or other conversion treatments as the first choice to improve tumor resectability. Therefore, studies should develop methods for accurately predicting LNM status for patients with GBC before making treatment decision.

Computed tomography (CT) is a widely used imaging method; however, it is limited in discovering positive LNM and the diagnostic rate is approximately 24\% (12). Most swollen LN caused probably by cholecystitis or biliary obstruction (13) can be detected through CT examination whereas positive LNs $<1 \mathrm{~mm}$ cannot be detected by the naked eye (14). Therefore, it is difficult for surgeons to distinguish LNM with the assistance of the conventional imaging method (15).

Radiomics technology, a product of artificial intelligence, can extract several imaging features from quantitative medical images $(16,17)$. Radiomics technology is a powerful tool for predicting LNM in different cancers (18-24). However, studies have not explored prediction of LNM based on radiomics technology in GBC.

The aim of the current study was to develop and validate a clinical-radiomics nomogram based on CT images that incorporate the radiomics signature and clinical pathological characteristics to quantitatively predict LNM of GBC.

\section{MATERIALS AND METHODS}

\section{Patients}

A total of 353 patients with GBC from two medical centers were enrolled to the current study. The training cohort and internal validation cohort comprised 209 patients and 47 patients with radical cholecystectomy recruited from the Second Affiliated Hospital Zhejiang University School of Medicine (Zhejiang, China) between January 2013 and December 2018, and between January 2019 and December 2020, respectively. The external validation cohort comprised 97 patients with radical cholecystectomy enrolled from the First Affiliated Hospital Zhejiang University School of Medicine (Zhejiang, China) between January 2013 and December 2018 following the same enrollment procedures. Inclusion criteria were as follows:
(1) pathologically confirmed GBC with an available histological report; (2) preoperative enhancement CT in abdomen performed within 1 month before surgery; (3) no chemotherapy or other treatment before operation; and (4) complete clinical and pathological data. Exclusion criteria were as follows: (1) had received any treatment (radiotherapy, chemotherapy, or immunotherapy) before CT examination; (2) patients that have undergone palliative surgery without lymphadenectomy; (3) lesions that cannot be identified in enhancement CT images; and (4) incomplete clinical data. A flowchart for patient recruitment is shown in Figure 1. The ethics committees of two hospitals approved this retrospective analysis and waived the requirement for informed consent.

Baseline clinical information, including age, gender, tumor markers, inflammatory indicators, presence of gallstones, symptoms, and CT report, was obtained from electronic medical records. Details on clinical characteristics are presented in Table 1. The gold standard for LNM was pathologically evaluated after surgery. Positive LNs were determined based on preoperative CT images by experienced radiologists, following the 8th AJCC TNM staging system. A flow diagram showing the study procedures is shown in Figure 2.

\section{Acquisition}

Abdominal CT enhancement examinations were performed on all patients within 1 month before the operation. Enhanced CT scan in the first hospital was performed using three CT scanners, including a 64-slice CT, a 256-slice CT (Philips Healthcare), and a 16-slice CT (Toshiba Medical System). Contrast-enhanced CT scan in the second hospital was performed using two CT scanners, including a 40-slice CT (Siemens AG) and a 320slice CT (Toshiba Medical Systems). CT scan parameters of the two hospitals were uniform and included the following: tube voltage at $120 \mathrm{kVp}$, tube current ranging from 125 to $300 \mathrm{mAs}$, pitch ranging from 0.6 to $1.25 \mathrm{~mm}$, slice thickness ranging from 3 to $5 \mathrm{~mm}$, and reconstruction interval from 3 to $5 \mathrm{~mm}$. A highpressure syringe was used to administer the non-ionic contrast agent Ultravist (Bayer Schering Pharma) $(1.5 \mathrm{ml} / \mathrm{kg}$ ) at a rate of $3.0 \mathrm{ml} / \mathrm{s}$. CT scans of the arterial phase and portal vein phase were performed at 25 to $35 \mathrm{~s}$ and 55 to $75 \mathrm{~s}$ after administration of the non-ionic contrast agent.

\section{Image Segmentation and Extraction of Features}

Regions of interest (ROIs) were manually segmented slice by slice around the lesions using an open-source imaging platform (ITK-SNAP, version 3.6.0). The portal venous phase was selected for image segmentation because it indicates the tumor boundary more accurately. Voxel size of the images was resampled to a normalized $1 \times 1 \times 1 \mathrm{~mm}^{3}$ to eliminate the pixel difference of the images, and voxel size and all the tumor regions were quantified as 64-gray levels to normalize the inhomogeneity of datasets due to variable tube voltages. Features were extracted from each segmented ROI and were divided into non-textual features and textural features using an in-house Python script (Pyradiomics version: stable; http://github.com/Radiomics/pyradiomics) (25). 
Patients with pathologically confirmed GBC from two hospitals were enrolled in this study

Inclusion criteria:

(1) pathologically confirmed GBC with an available histological report;;

(2) preoperative enhancement CT in abdomen performed within one month before surgery;

(3) no chemotherapy before operation;

(4) complete clinical and pathological data.
Exclusion criteria:

(1) had received any treatment before CT examination;

(2) palliative surgery without lymphadenectomy;

(3) the lesions not being identified in enhancement CT images;

(4) incomplete clinical data.

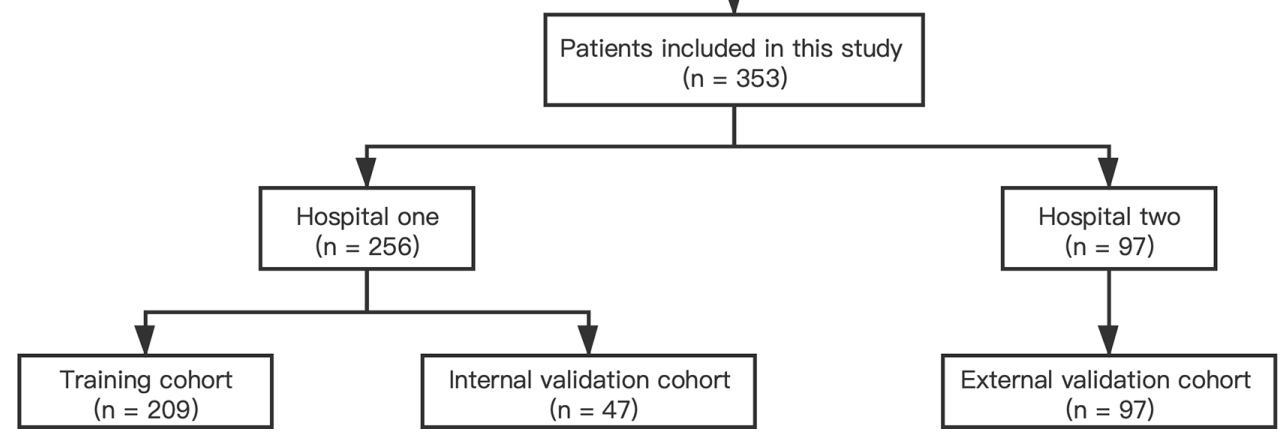

FIGURE 1 | Recruitment pathway of patients.

TABLE 1 | The clinical characteristics of the training cohort and the validation cohorts.

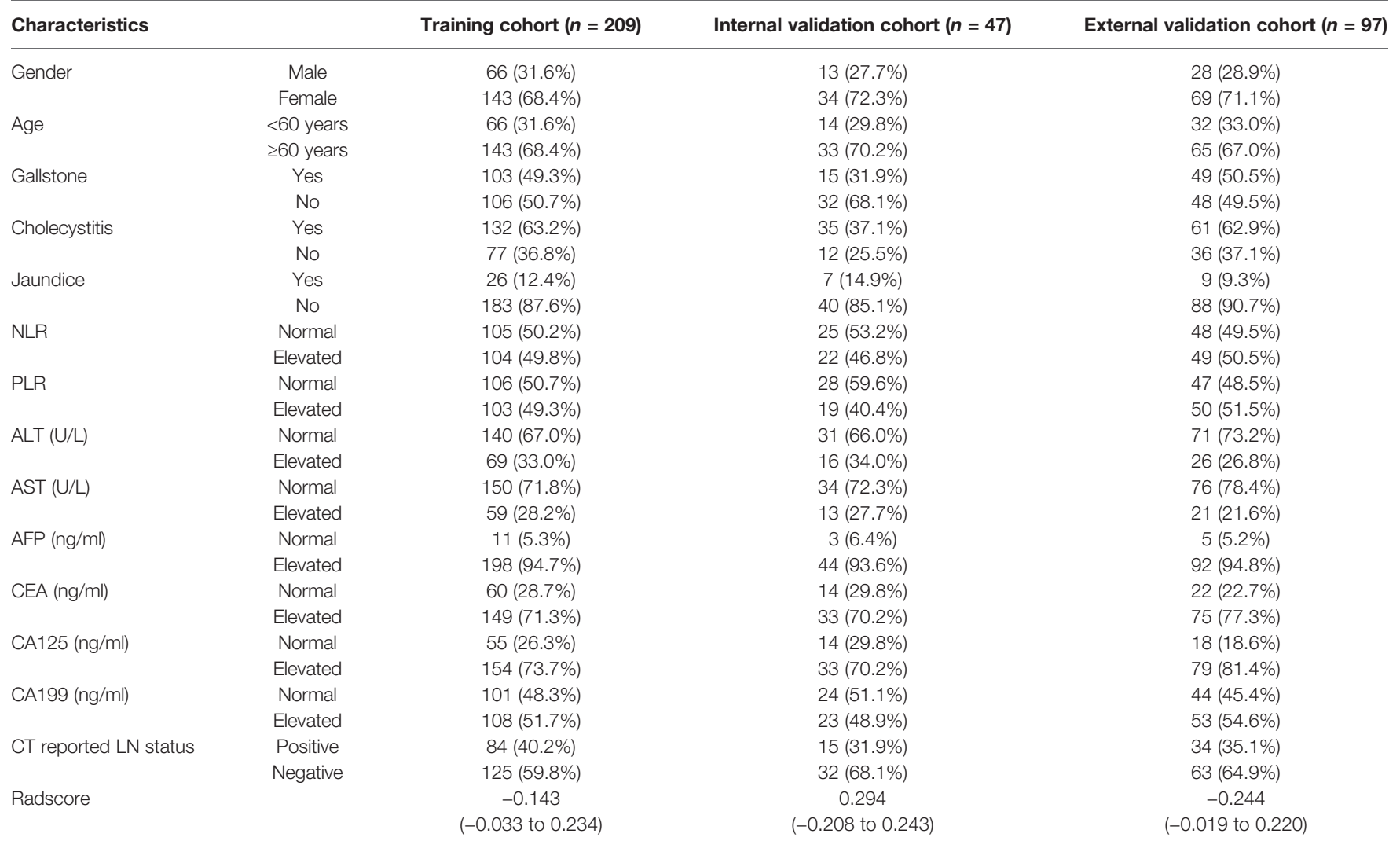

NLR, neutrophil-to-lymphocyte ratio; PLR, platelet-to-lymphocyte ratio; ALT, alanine aminotransferase; AST, aspartate aminotransferase; CEA, carcinoembryonic antigen; CA125, carbohydrate antigen 125; CA19-9, carbohydrate antigen 19-9. 


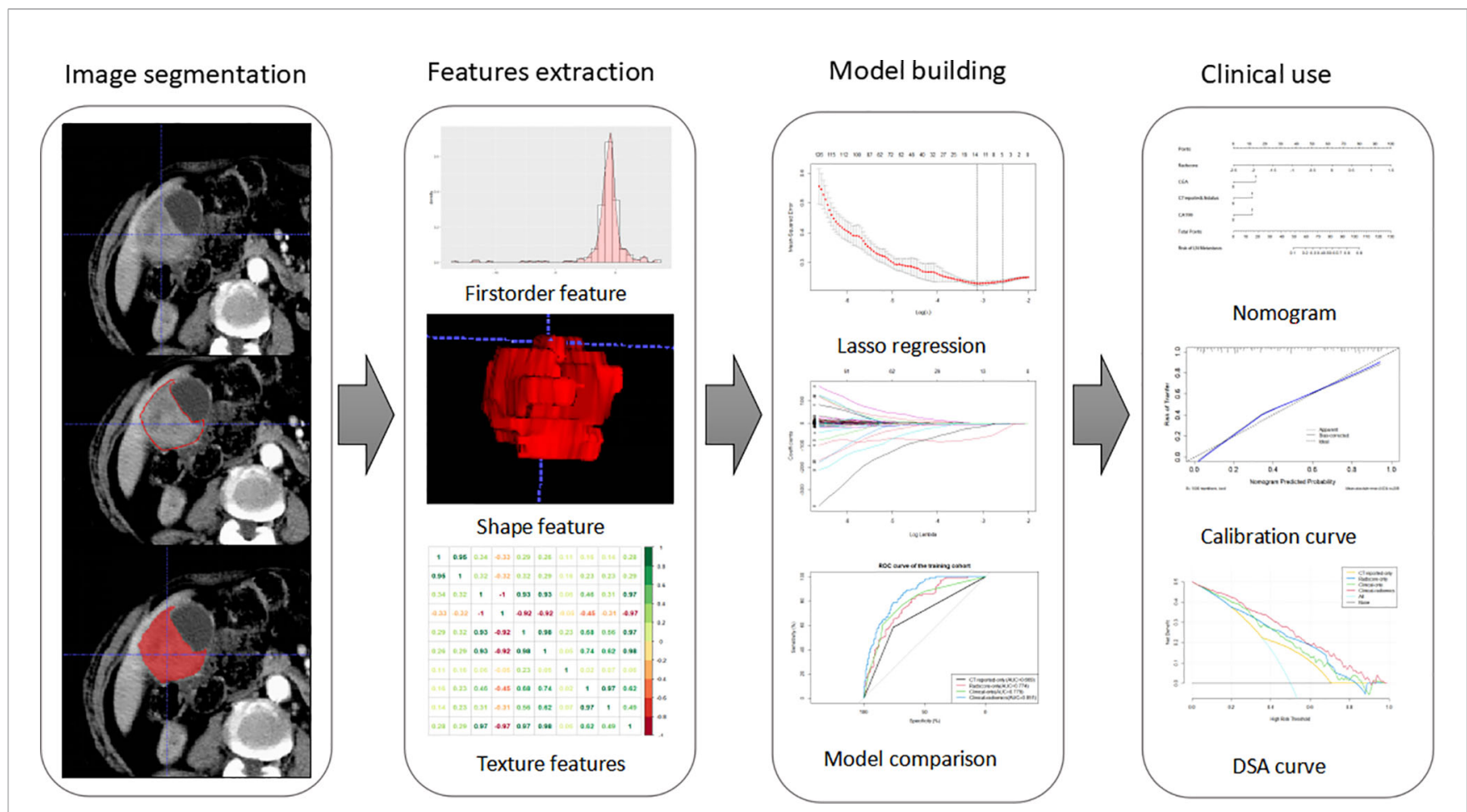

FIGURE 2 | Workflow of this study. Tumors are segmented manually on axial portal venous-phase CT section. Radiomics features were extracted from each CT images. LASSO regression was used to select radiomics features. The radiomics signature is constructed by a linear combination of selected features. Then, we built four models and compared their prediction performances with ROC curves. As a result, a clinical-radiomics nomogram was developed based on the best model. Calibration curves and DSA curves were used to evaluate the clinical utility of the nomogram.

Reproducibility of intra-observer and inter-observer agreement for ROI drawing was evaluated using 20 randomly chosen samples drawn from portal venous phase images by two radiologists blinded from patients' characteristics. A radiologist (reader 1) with 20 years of abdominal imaging experience and a surgeon (reader 2) with 15 years of surgical experience reviewed all CT scans to explore characteristics of each image. Reader 1 performed ROI drawing and feature extraction twice in a 2-week period, following a similar procedure to assess intra-observer reproducibility. In addition, reader 2 independently carried out the same procedure. Then, inter-observer agreement was assessed by comparing the results with the radiomics features extracted from the first ROI between two readers. Intra-observer and inter-observer agreement were assessed by intraclass correlation coefficient (ICC). An ICC > 0.75 represented satisfactory agreement.

\section{Radiomic Feature Selection and Radscore Building}

Least absolute shrinkage and selection operator (LASSO) algorithm was used to determine penalty coefficient with 10 -fold crossvalidation, which was then used to select optimal features from the training cohort $(26,27)$. A radiomics score (Radscore) of each patient was calculated by a linear combination of selected features, which were weighted based on their respective coefficients (Figure 3). More details on LASSO regression and radiomics features are presented in the Supplementary Material.

\section{Development of CT Reported-Only, Clinical, and Clinical-Radiomics Model}

Univariate logistic regression analysis was used to explore the relationship between LNM status and each clinical parameter, biomarkers, and Radscore in the training cohort. Significantly correlated clinical risk factors were then used for stepwise multivariate logistic regression analysis to build the clinicalonly model. CT-reported LN status, an independent risk factor, was used to build a CT reported-only model.

Moreover, clinical risk factors of the clinical-only model and Radscore were used for multiple logistic regression analysis to build the clinical-radiomics model.

\section{Model Comparison and Nomogram Development}

Each model was selected based on the minimal Akaike's information criterion (AIC) to determine the best diagnostic model. Area under the curve (AUC) was used to determine prediction accuracy of the four models in the training and validation cohort. Sensitivity, specificity, and accuracy of each model in the primary and validation cohort were calculated. A nomogram was then built based on the most effective model for LNM prediction of GBC. A calibration curve was plotted to evaluate both discrimination and calibration of the best nomogram. 


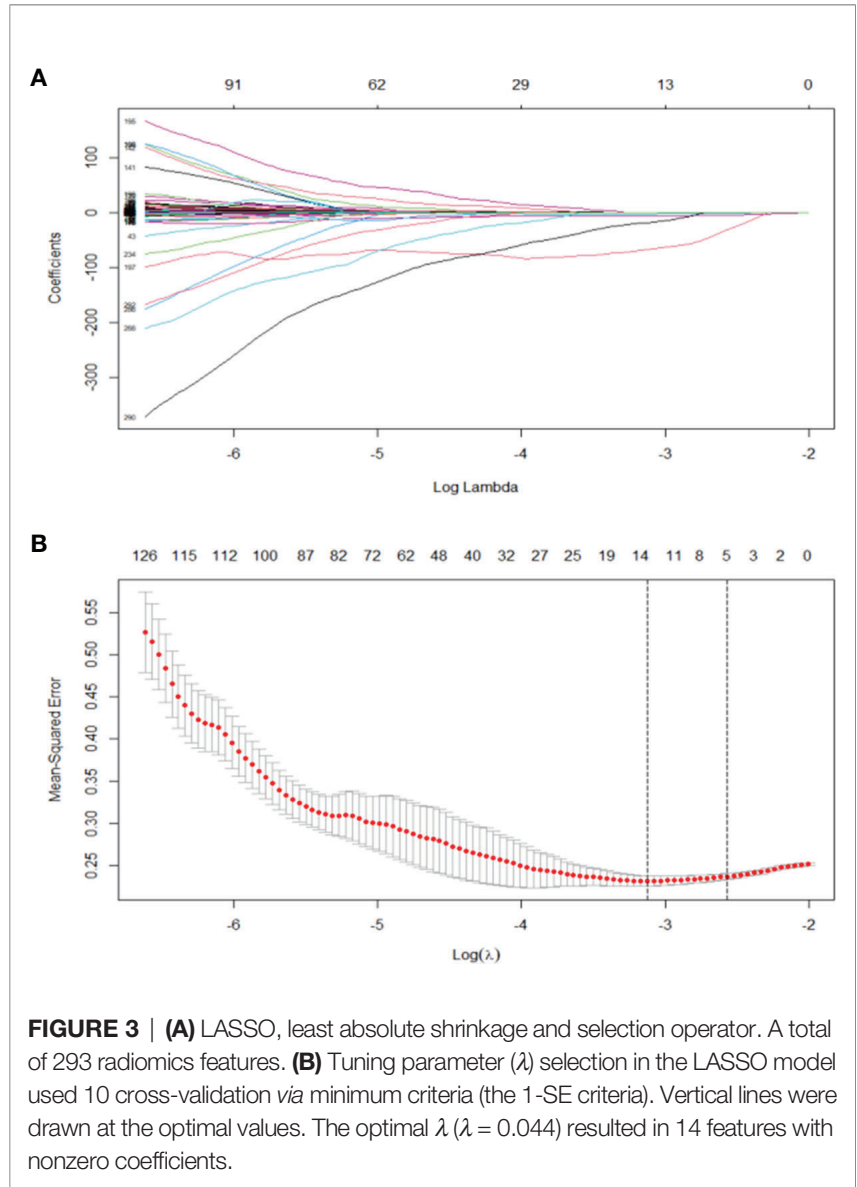

\section{Clinical Use of the Nomogram}

To explore the clinical utility of the nomogram, decision curve analysis (DCA) was performed based on four models to determine the utility of the nomogram for a range of threshold probabilities.

\section{Statistical Analysis}

Statistical analyses were conducted using SPSS software (version 21.0) and $\mathrm{R}$ software (version 4.0.0). Continuous variables were compared using Mann-Whitney $U$ test, whereas category variables were compared using Chi-squared or Fisher exact tests. Univariate and multivariate Cox regression analyses were performed to determine predictors of LN. Variables with $p$-value $<0.05$ in univariate analysis were selected for multivariate analysis. LASSO regression analysis was performed using the "glmnet" package in R software version 4.0.0. "pROC" package was used to plot the ROC curve. Nomogram construction and calibration plots were generated using "rms" package in R. DCA was performed using the "dca.R" package. A two-sided $p<0.05$ was considered statistically significant.

\section{RESULTS}

\section{Clinical Characteristics}

Patient characteristics in the training and validation cohorts are presented in Table 1. Analysis showed no significant differences in clinicopathological characteristics between the three groups.

\section{Feature Selection and Radscore Development}

A total of 293 radiomics features based on the training cohort were reduced to 14 potential predictors using LASSO regression analysis (Figure 2). A radiomics score (Radscore) was then calculated using the formula presented in the Supplementary material. Findings from univariate logistic regression analysis showed that the Radscore (OR $=9.610$; 95\% CI: 4.579-20.168, $p<0.001)$ was an independent variable for prediction of LNM in the training cohort (Table 2).

\section{Development of the Clinical Model and CT Reported-Only Model}

Results of the univariate analysis and the multivariate regression analysis using training cohort are shown in Table 2. Univariate analysis of the training cohort identified NLR, PLR, aspartate aminotransferase (AST), carcinoembryonic antigen (CEA), carcinoembryonic antigen 125 (CA125), carcinoembryonic antigen 199 (CA199), and CT reported LN status as statistically significant risk factors $(p<0.05)$ (Table 2 ). Statistically significant variables selected from the univariate analysis were used for binary multiple logistic regression, and the findings showed that CEA (OR $=2.898 ; 95 \% \mathrm{CI}: 1.418-5.921$; $p=0.004)$, CA199 (OR $=3.597 ; 95 \%$ CI: $1.921-6.733 ; p<0.001)$, and CT reported $\mathrm{LN}$ status $(\mathrm{OR}=2.962 ; 95 \%$ CI: 1.557-5.635; $p=0.001$ ) were independent risk factors of LNM in the training cohort. The three independent risk factors based on the logistic multivariate regression analysis were used for construction of the clinical model (Table 3). In addition, CT reported LN status identified as an independent variable in univariate analysis was used to build CT reported-only model $(\mathrm{OR}=4.325 ; 95 \% \mathrm{CI}$ : 2.396-7.809; $p<0.001)$.

\section{Development of the Clinical- Radiomics Model}

Eight factors, namely, NLR, PLR, AST, CEA, CA125, CA199, CT reported LN status, and Radscore, were used for binary multivariate logistic regression analysis (Table 2). The findings showed that CEA (OR $=3.122 ; 95 \%$ CI: $1.426-6.835 ; p=0.004)$, CA199 (OR $=2.592 ; 95 \%$ CI: $1.288-5.215 ; p=0.008)$, CT reported LN status $(\mathrm{OR}=2.597 ; 95 \%$ CI: $1.278-5.279$; $p=0.008$ ), and Radscore (OR $=7.415$; 95\% CI: 3.384-16.246; $p<0.001)$ were significant predictors of LNM; thus, they were used to build a clinical-radiomics model for LNM (Table 3). Notably, Radscore was the dominant factor affecting prediction of LNM in the clinical-radiomics model.

\section{Model Comparison and Validation of the Nomogram}

AIC was used to determine the goodness of model fitting. Comparison was performed for the clinical model (AIC = 242.27), the Radscore-only model ( $\mathrm{AIC}=237.31$ ), the CT reported-only model (AIC $=267.68$ ), and the clinical-radiomics model $($ AIC $=209.18)$. Notably, the clinical-radiomics model had the lowest AIC value (AIC $=209.18)$ and was identified as the best model. 
TABLE 2 | Univariate and multivariate logistic regression analysis for LN metastasis in the primary cohort.

\begin{tabular}{|c|c|c|c|c|c|c|}
\hline \multirow[t]{3}{*}{ Characteristics } & \multicolumn{2}{|c|}{ Univariate analysis } & \multicolumn{4}{|c|}{ Multivariate analysis } \\
\hline & \multirow[b]{2}{*}{ OR (95\% Cl) } & \multirow[b]{2}{*}{$P$} & \multicolumn{2}{|c|}{ Clinical model } & \multicolumn{2}{|c|}{ Clinical-radiomics model } \\
\hline & & & OR $(95 \% \mathrm{Cl})$ & $\boldsymbol{P}$ & OR $(95 \% \mathrm{Cl})$ & $p$ \\
\hline Gender & $1.301(0.723-2.343)$ & 0.380 & NA & NA & NA & NA \\
\hline Age & $0.762(0.425-1.368)$ & 0.363 & NA & NA & NA & NA \\
\hline Gallstone & $0.776(0.450-1.337)$ & 0.361 & NA & NA & NA & NA \\
\hline Cholecystitis & $0.929(0.529-1.631)$ & 0.797 & NA & NA & NA & NA \\
\hline Jaundice & $1.643(0.716-3.770)$ & 0.241 & NA & NA & NA & NA \\
\hline NLR & 2.138 (1.229-3.718) & 0.007 & 1.207 (0.590-2.467) & 0.607 & $1.044(0.484-2.252)$ & 0.913 \\
\hline PLR & $2.622(1.498-4.590)$ & 0.001 & $1.963(0.977-3.944)$ & 0.058 & $1.888(0.900-3.962)$ & 0.093 \\
\hline $\mathrm{ALT}$ & 1.497 (0.839-2.672) & 0.172 & NA & NA & NA & NA \\
\hline AST & $2.014(1.092-3.715)$ & 0.025 & 1.403 (0.680-2.897) & 0.360 & 1.299 (0.579-2.912) & 0.525 \\
\hline AFP & $3.200(0.825-12.419)$ & 0.093 & NA & NA & NA & NA \\
\hline CEA & $4.323(2.251-8.303)$ & $<0.001$ & $2.898(1.418-5.921)$ & 0.004 & 3.183 (1.423-7.123) & 0.005 \\
\hline CA125 & 3.925 (2.016-7.643) & $<0.001$ & $1.872(0.857-4.087)$ & 0.116 & $1.557(0.661-3.672)$ & 0.311 \\
\hline CA199 & 4.895 (2.722-8.800) & $<0.001$ & $3.597(1.921-6.733)$ & 0.001 & $2.230(1.074-4.632)$ & 0.031 \\
\hline CT reported LN status & 4.325 (2.396-7.809) & $<0.001$ & $2.962(1.557-5.635)$ & 0.010 & $2.261(1.075-4.755)$ & 0.031 \\
\hline Radscore & $9.610(4.579-20.168)$ & $<0.001$ & NA & NA & 6.645 (3.025-14.597) & $<0.001$ \\
\hline
\end{tabular}

$O R$, odds ratio; NLR, neutrophil-to-lymphocyte ratio; PLR, platelet-to-lymphocyte ratio; $A L T$, alanine aminotransferase; $A S T$, aspartate aminotransferase; CEA, carcinoembryonic antigen; CA125, carbohydrate antigen 125; CA19-9, carbohydrate antigen 19-9, NA, not available.

ROC curves were used to evaluate the accuracy and predictive value of the four models (Table 4 and Figure 5A). In the training cohort, the clinical-radiomics model showed the highest discrimination between LN positive and negative cases, with an AUC of 0.851 (95\% CI, 0.801-0.901). The AUC value of the clinicalradiomics model was significantly higher compared with that of the clinical model (AUC 0.779; 95\% CI, 0.715-0.842; $p=0.001$ ), Radscore-only model (AUC 0.774; 95\% CI, 0.712-0.836; $p=$ 0.001 ), and CT reported-only model (AUC 0.669 ; $95 \%$ CI, $0.595-$ $0.743 ; p<0.001)$. In the internal validation cohort and external validation cohort, the radiomics model showed the highest AUC of 0.824 (95\% CI, 0.741-0.908) and 0.819 (95\% CI, 0.645-0.993), respectively. The clinical-radiomics model showed the best accuracy for prediction efficiency of LNM in the training cohort (sensitivity: $86.7 \%$; specificity: 67.6\%; accuracy: $78.0 \%$ ), internal validation cohort (sensitivity: $73.3 \%$; specificity: $100 \%$; accuracy: $91.5 \%$ ), and external validation cohort (sensitivity: $78.7 \%$; specificity: 80\%; accuracy: 79.4\%) (Table 4).

The clinical-radiomics model showed the best discrimination and predictive ability among the four models. Therefore, a clinical-radiomics nomogram was successfully developed based on the clinical-radiomics model (Figure 4). A calibration curve of the clinical-radiomics nomogram for the probability of LNM showed good consistency between prediction and actual LN status in the three cohorts (Figure 5B).

\section{Clinical Application}

DCA curves for the clinical-radiomics model, the clinical model, the Radscore-only model, and the CT reported-only model in both the training and validation cohorts are shown in Figure 5C. The threshold probability of the clinical-radiomics nomogram was more than $10 \%$, which was better compared with the other three models in predicting LNM. The combined nomogram including radiomics signature showed the maximum clinical utility at almost all threshold probabilities.

\section{DISCUSSION}

To the best of our knowledge, this is the first study to develop a clinical-radiomics nomogram based on radiomics technology for preoperative prediction of LNM in patients with GBC. The clinical-radiomics model incorporated radiomics signature and three clinical variables including CEA, CA199, and CT reported LN status. The Radscore was calculated based on the radiomics signature and the findings showed that it was an independent factor in predicting LNM. Addition of radiomic analysis significantly improved the predictive accuracy of the combined model. The findings indicated that the clinical-radiomics nomogram is effective for preoperative prediction of LNM in GBC and can help in making clinical decisions during GBC treatment.

Previous studies explored non-invasive methods that can quantitatively predict preoperative LNM in GBC. Several conventional imaging examinations such as contrast-enhanced CT, MRI, and 18-FDG PET/CT are used to determine the LN status and LN larger than $1 \mathrm{~cm}$ in diameter is considered a standard for positive LNM in these examinations. However, swollen LN can be caused by biliary inflammation or biliary obstruction. Petrowsky et al. reported that the accuracy of enhanced CT and PET/CT for regional LNM prediction was $24 \%$ vs. $12 \%$ (12). A meta-analysis of 14 institutes reported that although MRI is effective in predicting LNM of GBC, it is challenging to detect LNM less than $1 \mathrm{~cm}$ (28). Fine needle aspiration for pathological biopsy is the gold standard for preoperative diagnosis; however, it can only be applied to a limited range of patients. The method is associated with severe complications such as bleeding, tumor dissemination, and lymphatic fistula owing to the use of fine needle aspiration. Several studies report that inflammation and metabolites in tumor areas of the biliary system may cause LN hyperplasia (29-31). Therefore, the current study established a clinical model 


\begin{tabular}{|c|c|c|c|c|c|c|c|c|c|c|c|c|}
\hline \multirow[t]{2}{*}{ Variable } & \multicolumn{3}{|c|}{ CT reported-only model } & \multicolumn{3}{|c|}{ Radscore-only model } & \multicolumn{3}{|c|}{ Clinical model } & \multicolumn{3}{|c|}{ Clinical-radiomics model } \\
\hline & OR $(95 \% \mathrm{Cl})$ & $P$ & AIC & OR (95\% Cl) & $P$ & AIC & OR $(95 \% \mathrm{Cl})$ & $\boldsymbol{P}$ & AIC & OR (95\% Cl) & $p$ & AIC \\
\hline CEA & NA & NA & 267.68 & NA & NA & 237.31 & $2.898(1.418-5.921)$ & 0.004 & 242.27 & $3.122(1.426-6.835)$ & 0.004 & 209.18 \\
\hline CA199 & NA & NA & & NA & NA & & $3.597(1.921-6.733)$ & $<0.001$ & & $2.592(1.288-5.215)$ & 0.008 & \\
\hline CT reported LN status & $4.325(2.396-7.809)$ & $<0.001$ & & NA & NA & & $2.962(1.557-5.635)$ & 0.001 & & $2.597(1.278-5.279)$ & 0.008 & \\
\hline Radscore & NA & NA & & $9.610(4.579-20.168)$ & $<0.001$ & & NA & NA & & $7.415(3.384-16.246)$ & $<0.001$ & \\
\hline
\end{tabular}

AIC, Akaike's information criterion; $\mathrm{OR}$, odds ratio; $\mathrm{Cl}$, confidence interval, NA, not available.

TABLE 4 | Accuracy and predictive value between four models.

\begin{tabular}{|c|c|c|c|c|c|c|c|c|c|c|c|c|}
\hline \multirow[t]{2}{*}{ Models } & \multicolumn{4}{|c|}{ Training cohort } & \multicolumn{4}{|c|}{ Internal validation cohort } & \multicolumn{4}{|c|}{ External validation cohort } \\
\hline & ensitivity & ecificity & curacy & CI) & nsitivity & ecificity & cy & AUC & ensitivity & pecificity & Accuracy & AUC $(95 \% \mathrm{Cl})$ \\
\hline $\begin{array}{l}\text { CT reported-only } \\
\text { Radscore-only } \\
\text { Clinical } \\
\text { Clinical-radiomics }\end{array}$ & $\begin{array}{l}(57 / 98) 58.2 \% \\
(80 / 98) 81.6 \% \\
(75 / 98) 76.5 \% \\
(85 / 98) 86.7 \%\end{array}$ & $\begin{array}{l}(84 / 111) 75.7 \% \\
(67 / 111) 60.4 \% \\
(76 / 111) 68.5 \% \\
(75 / 111) 67.6 \%\end{array}$ & $\begin{array}{l}(141 / 209) 67.5 \% \\
(147 / 209) 70.3 \% \\
(151 / 209) 72.2 \% \\
(160 / 209) 78.0 \%\end{array}$ & $\begin{array}{l}0.669(0.595-0.743) \\
0.774(0.712-0.836) \\
0.779(0.715-0.842) \\
0.851(0.801-0.901)\end{array}$ & $\begin{array}{c}(6 / 15) 40 \% \\
(12 / 15) 80.0 \% \\
(12 / 15) 80.0 \% \\
(11 / 15) 73.3 \%\end{array}$ & $\begin{array}{l}(23 / 32) 71.9 \% \\
(30 / 32) 93.8 \% \\
(18 / 32) 56.2 \% \\
(32 / 32) 100 \%\end{array}$ & $\begin{array}{l}(29 / 47) 61.7 \% \\
(42 / 47) 89.4 \% \\
(30 / 47) 68.1 \% \\
(77 / 97) 91.5 \%\end{array}$ & $\begin{array}{l}0.559(0.379-0.739) \\
0.763(0.668-0.857) \\
0.731(0.631-0.832) \\
0.819(0.645-0.993)\end{array}$ & $\begin{array}{l}(26 / 47) 55.3 \% \\
(27 / 47) 57.4 \% \\
(37 / 47) 78.7 \% \\
(37 / 47) 78.7 \%\end{array}$ & $\begin{array}{l}(42 / 50) 84.0 \% \\
(42 / 50) 84.0 \% \\
(31 / 50) 62.0 \% \\
(40 / 50) 80 \%\end{array}$ & $\begin{array}{l}(68 / 97) 70.1 \% \\
(69 / 97) 71.1 \% \\
(68 / 97) 70.1 \% \\
(77 / 97) 79.4 \%\end{array}$ & $\begin{array}{l}0.697(0.590-0.803) \\
0.763(0.668-0.857) \\
0.731(0.631-0.832) \\
0.824(0.741-0.908)\end{array}$ \\
\hline
\end{tabular}

AUC, area under the curve. 


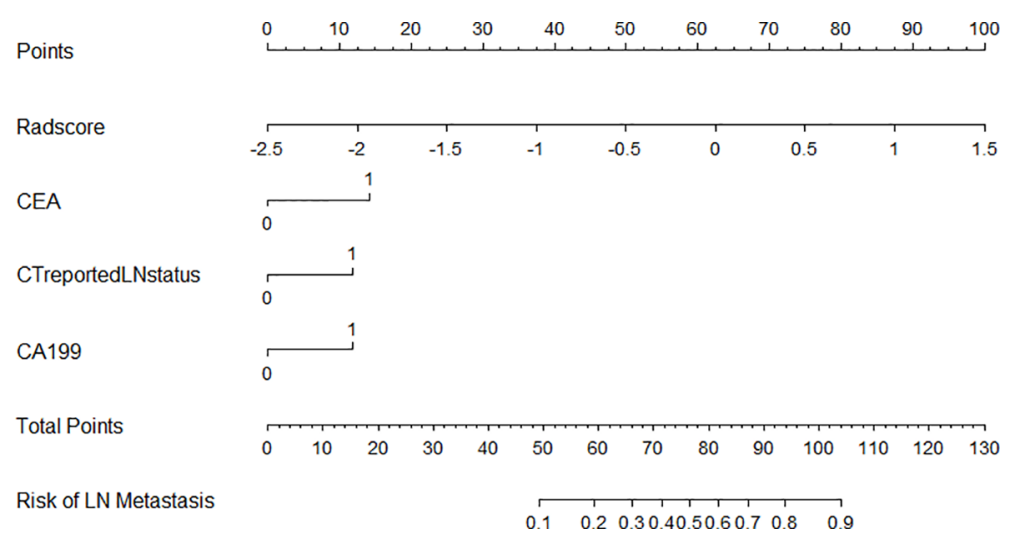

FIGURE 4 | Developed clinical-radiomics nomogram. The clinical-radiomics nomogram was developed including Radscore, CEA, CA199, and CT reported LN status in the training group.

A

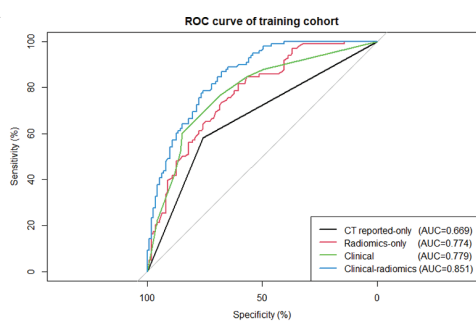

B

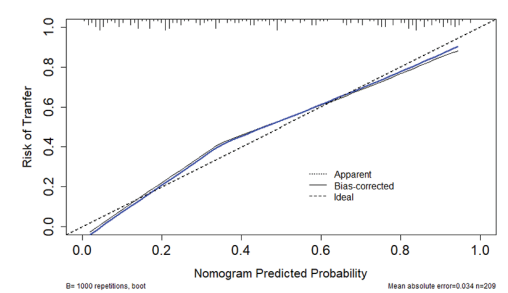

C

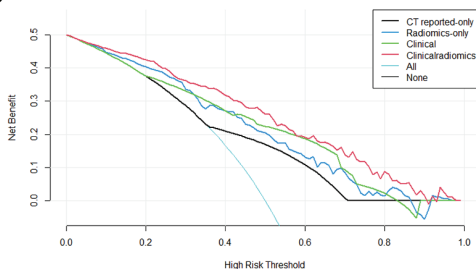

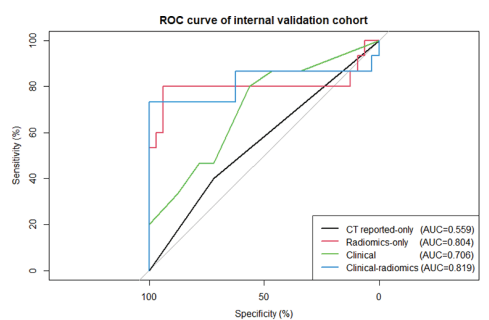
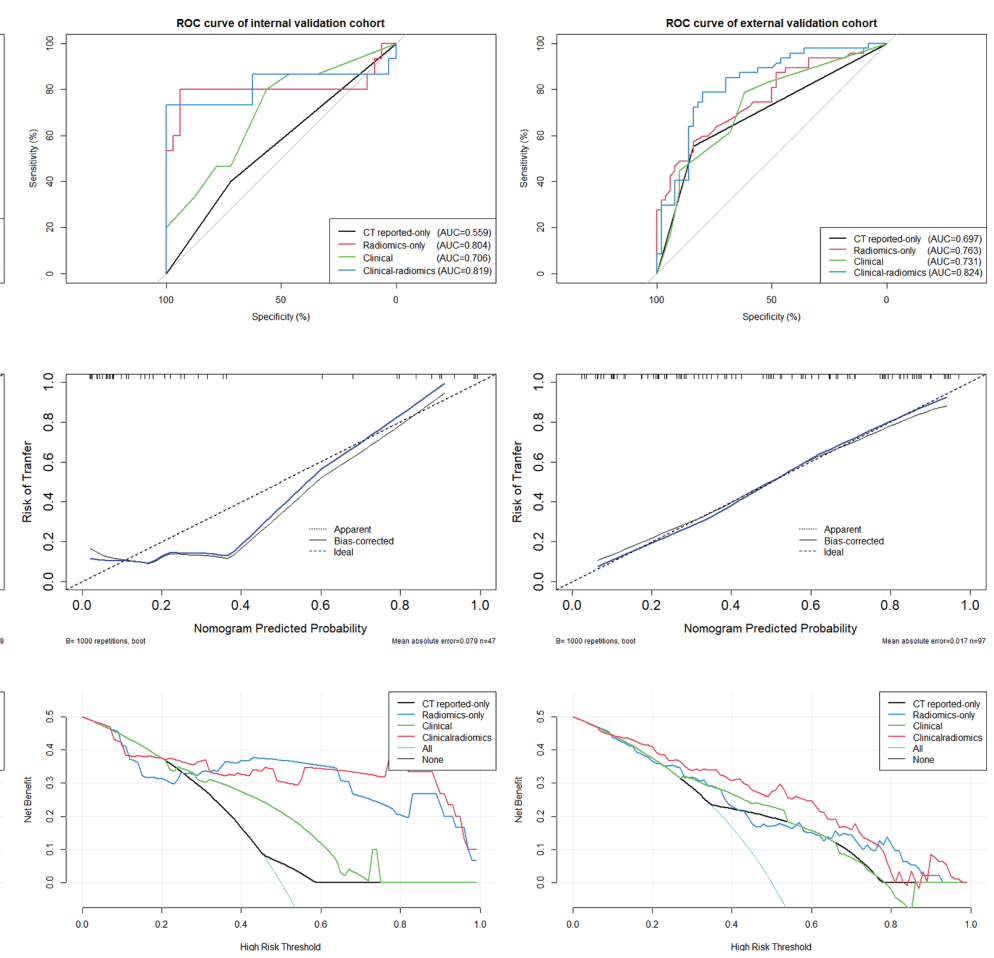

FIGURE 5 | (A) ROC curves of the clinical-radiomics model, the clinical model, the Radscore-only model, and the CT reported-only model were shown in the training cohort, internal validation cohort, and external validation cohort, respectively. (B) Calibration curves of the clinical-radiomics nomogram for predicting LNM between prediction and actual $\mathrm{LN}$ status in the training cohort, internal validation cohort, and external validation cohort. The $45^{\circ}$ straight line represents an ideal model perfectly calibrated with an outcome. A closer distance between two curves indicates higher accuracy. (C) Decision curve analysis for the clinical-radiomics nomogram, the clinical model, the Radscore-only model, and the CT reported-only model in both three cohorts. The $y$-axis measures the net benefit. The red line represents the clinical-radiomics nomogram. The blue line represents the Radscore-only model. The green line represents the clinical model. The yellow line represents CT reported-only model. The azure line represents the assumption that all patients had LNM. The black line represents the assumption that no patients had LNM.

including multiple parameters for comparison. The findings showed that NLR, PLR, AST, and CA125 were independent predictive factors for LNM in univariate analysis. However, analysis showed no significant correlation between these markers with LNM using multivariate logistics regression analysis. The clinical model build using CEA, CA199, and CT reported LN status showed higher prediction value compared with CT reported-only model; however, its overall accuracy was 
still unsatisfactory. Conventional imaging methods are based on morphological criteria and serum biomarkers and do not meet the clinical need for quantitative and accurate diagnosis.

On the contrary, radiomics technology is a quantitative method and thus it is effective in preoperative LN assessment. Several studies report that radiomics can be correlated with tumor gene characteristics and protein phenotypes to predict the biological behavior of tumors $(32,33)$. Metastatic LN and non-metastatic LN present different biological behaviors (10). Studies report that a model established based on radiomics has great potential in predicting LNM of malignant tumors (21-23). In the current study, a CT-based radiomics model composed of 14 radiomics signatures was established using LASSO regression. The Radscore established included shape, first-order, and textural features. Radiomics analysis of CT images can help distinguish between positive LNM and negative LNM for gastric cancer and colorectal cancer $(20,21)$. The findings of the current study showed that the radiomics model based on morphological features and texture features has better predictive accuracy and goodness of fit for LNM compared with the single CT reported-only model (AUC = 0.781 vs. AUC = 0.669; AIC 237.31 to 267.68).

For better clinical application, a clinical-radiomics nomogram was established that integrated Radscore and clinical variables. This comprehensive nomogram showed higher accuracy and discrimination of the LNM in GBC compared with the other three models. Calibration curves and DCA curves showed that the nomogram had high consistency and potential clinical applicability in the two medical centers. The clinical-radiomics nomogram can be used effectively to determine the possibility of surgical R0 resection, thus assisting in preoperative treatment decision-making. GBC patients suspected of positive LNM based on conventional imaging reports can use the nomogram to reconfirm their LN status. In addition, surgeons can use the nomogram to accurately assess the necessity for LN resection before surgery to benefit patients with actual negative LNM, thus reducing complications and hospitalization costs.

The current study had some limitations. Firstly, genetic diagnosis related to progress of GBC may provide more value in the diagnosis of LNM through development of radiogenomic biomarkers. Further studies that include genotypes to new predictive models to improve the model's diagnostic accuracy should be conducted. Secondly, the data used to build the model in the current study were obtained from two large-scale medical centers in a region that may lead to data bias. Therefore, studies

\section{REFERENCES}

1. Hundal R, Shaffer EA. Gallbladder Cancer: Epidemiology and Outcome. Clin Epidemiol (2014) 6:99-109. doi: 10.2147/CLEP.S37357

2. Randi G, Franceschi S, La Vecchia C. Gallbladder Cancer Worldwide: Geographical Distribution and Risk Factors. Int J Cancer (2006) 118 (7):1591-602. doi: 10.1002/ijc.21683

3. Rawla P, Sunkara T, Thandra KC, Barsouk A. Epidemiology of Gallbladder Cancer. Clin Exp Hepatol (2019) 5(2):93-102. doi: 10.5114/ceh.2019.85166

4. Duffy A, Capanu M, Abou-Alfa GK, Huitzil D, Jarnagin W, Fong Y, et al. Gallbladder Cancer (GBC): 10-Year Experience at Memorial Sloan-Kettering Cancer Centre (MSKCC). J Surg Oncol (2008) 98(7):485-9. doi: 10.1002/jso.21141 should include more patients from multiple centers as a verification cohort to verify the clinical applicability and robustness of the nomogram.

In summary, the clinical-radiomics nomogram reported in the current study can be used as a non-invasive biomarker for preoperative prediction of LNM in GBC patients. The findings show that the model is useful in clinical decision-making and can improve the survival outcome of patients with GBC.

\section{DATA AVAILABILITY STATEMENT}

The raw data supporting the conclusions of this article will be made available by the authors, without undue reservation.

\section{ETHICS STATEMENT}

The ethics committees of two hospitals approved this retrospective analysis and waived the requirement for informed consent.

\section{AUTHOR CONTRIBUTIONS}

XLiu collected CT images and clinical data, and drafted the original manuscript. XLia analyzed the data and revised the manuscript. LR and SY provided the idea, designed the research, segmented the images, and revised the manuscript. All authors contributed to the article and approved the submitted version.

\section{FUNDING}

This project was supported by the National Natural Science Foundation of China (No. 81572975), the Key Research and Development Project of the Science and Technology Department of Zhejiang, China (No. 2015C03053), and the Zhejiang Provincial Program for the Cultivation of High-level Innovative Health talents.

\section{SUPPLEMENTARY MATERIAL}

The Supplementary Material for this article can be found online at: https://www.frontiersin.org/articles/10.3389/fonc.2021. 633852/full\#supplementary-material

5. Tran TB, Nissen NN. Surgery for Gallbladder Cancer in the US: A Need for Greater Lymph Node Clearance. J Gastrointest Oncol (2015) 6(5):452-8. doi: 10.3978/j.issn.2078-6891.2015.062

6. Sung YN, Song M, Lee JH, Song KB, Hwang DW, Ahn CS, et al. Validation of the 8th Edition of the American Joint Committee on Cancer Staging System for Gallbladder Cancer and Implications for the Follow-Up of Patients Without Node Dissection. Cancer Res Treat (2020) 52(2):455-68. doi: 10.4143/crt.2019.271

7. Fairweather M, Balachandran VP, D'Angelica MI. Surgical Management of Biliary Tract Cancers. Chin Clin Oncol (2016) 5(5):63. doi: 10.21037/cco.2016.10.03

8. Negi SS, Singh A, Chaudhary A. Lymph Nodal Involvement as Prognostic Factor in Gallbladder Cancer: Location, Count or Ratio? J Gastrointest Surg (2011) 15(6):1017-25. doi: 10.1007/s11605-011-1528-4 
9. Bridgewater J, Galle PR, Khan SA, Llovet JM, Park JW, Patel T, et al. Guidelines for the Diagnosis and Management of Intrahepatic Cholangiocarcinoma. J Hepatol (2014) 60(6):1268-89. doi: 10.1016/j.jhep.2014.01.021

10. Shirai Y, Sakata J, Wakai T, Ohashi T, Ajioka Y, Hatakeyama K. Assessment of Lymph Node Status in Gallbladder Cancer: Location, Number, or Ratio of Positive Nodes. World J Surg Oncol (2012) 10:87. doi: 10.1186/1477-7819-10-87

11. Ito $\mathrm{H}$, Ito $\mathrm{K}$, D’Angelica $\mathrm{M}$, Gonen $\mathrm{M}$, Klimstra D, Allen $\mathrm{P}$, et al. Accurate Staging for Gallbladder Cancer: Implications for Surgical Therapy and Pathological Assessment. Ann Surg (2011) 254(2):320-5. doi: 10.1097/ SLA.0b013e31822238d8

12. Petrowsky H, Wildbrett P, Husarik DB, Hany TF, Tam S, Jochum W, et al. Impact of Integrated Positron Emission Tomography and Computed Tomography on Staging and Management of Gallbladder Cancer and Cholangiocarcinoma. J Hepatol (2006) 45(1):43-50. doi: 10.1016/ j.jhep.2006.03.009

13. de Savornin Lohman EAJ, de Bitter TJJ, van Laarhoven CJHM, Hermans JJ, de Haas RJ, de Reuver PR. The Diagnostic Accuracy of CT and MRI for the Detection of Lymph Node Metastases in Gallbladder Cancer: A Systematic Review and Meta-Analysis. Eur J Radiol (2019) 110:156-62. doi: 10.1016/ j.ejrad.2018.11.034

14. Kalra N, Suri S, Gupta R, Natarajan SK, Khandelwal N, Wig JD, et al. MDCT in the Staging of Gallbladder Carcinoma. AJR Am J Roentgenol (2006) 186 (3):758-62. doi: 10.2214/AJR.04.1342

15. Jiang F, Jiang Y, Zhi H, Dong Y, Li H, Ma S, et al. Artificial Intelligence in Healthcare: Past, Present and Future. Stroke Vasc Neurol (2017) 2(4):230-43. doi: 10.1136/svn-2017-000101

16. Lambin P, Leijenaar RTH, Deist TM, Peerlings J, de Jong EEC, van Timmeren J, et al. Radiomics: The Bridge Between Medical Imaging and Personalized Medicine. Nat Rev Clin Oncol (2017) 14(12):749-62. doi: 10.1038/ nrclinonc.2017.141

17. Lambin P, Rios-Velazquez E, Leijenaar R, Carvalho S, van Stiphout RG, Granton P, et al. Radiomics: Extracting More Information From Medical Images Using Advanced Feature Analysis. Eur J Cancer (2012) 48(4):441-6. doi: 10.1016/j.ejca.2011.11.036

18. Han L, Zhu Y, Liu Z, Yu T, He C, Jiang W, et al. Radiomic Nomogram for Prediction of Axillary Lymph Node Metastasis in Breast Cancer. Eur Radiol (2019) 29(7):3820-9. doi: 10.1007/s00330-018-5981-2

19. Wu S, Zheng J, Li Y, Yu H, Shi S, Xie W, et al. A Radiomics Nomogram for the Preoperative Prediction of Lymph Node Metastasis in Bladder Cancer. Clin Cancer Res (2017) 23(22):6904-11. doi: 10.1158/1078-0432.CCR-17-1510

20. Huang YQ, Liang CH, He L, Tian J, Liang CS, Chen X, et al. Development and Validation of a Radiomics Nomogram for Preoperative Prediction of Lymph Node Metastasis in Colorectal Cancer. J Clin Oncol (2016) 34(18):2157-64. doi: 10.1200/JCO.2015.65.9128

21. Dong D, Fang MJ, Tang L, Shan XH, Gao JB, Giganti F, et al. Deep Learning Radiomic Nomogram Can Predict the Number of Lymph Node Metastasis in Locally Advanced Gastric Cancer: An International Multicenter Study. Ann Oncol (2020) 31(7):912-20. doi: 10.1016/j.annonc.2020.04.003

22. Jiang Y, Wang W, Chen C, Zhang X, Zha X, Lv W, et al. Radiomics Signature on Computed Tomography Imaging: Association With Lymph Node Metastasis in Patients With Gastric Cancer. Front Oncol (2019) 9:340. doi: $10.3389 /$ fonc. 2019.00340

23. Li J, Dong D, Fang M, Wang R, Tian J, Li H, et al. Dual-Energy CT-Based Deep Learning Radiomics Can Improve Lymph Node Metastasis Risk
Prediction for Gastric Cancer. Eur Radiol (2020) 30(4):2324-33. doi: 10.1007/s00330-019-06621-x

24. Ji GW, Zhu FP, Zhang YD, Liu XS, Wu FY, Wang K, et al. A Radiomics Approach to Predict Lymph Node Metastasis and Clinical Outcome of Intrahepatic Cholangiocarcinoma. Eur Radiol (2019) 29(7):3725-35. doi: 10.1007/s00330-019-06142-7

25. van Griethuysen JJM, Fedorov A, Parmar C, Hosny A, Aucoin N, Narayan V, et al. Computational Radiomics System to Decode the Radiographic Phenotype. Cancer Res (2017) 77(21):e104-7. doi: 10.1158/0008-5472.CAN-17-0339

26. Tibshirani R. The Lasso Method for Variable Selection in the Cox Model. Stat Med (1997) 16(4):385-95. doi: 10.1002/(sici)1097-0258(19970228)16:4<385:: aid-sim380 $>3.0 . \operatorname{co} ; 2-3$

27. Sauerbrei W, Royston P, Binder H. Selection of Important Variables and Determination of Functional Form for Continuous Predictors in Multivariable Model Building. Stat Med (2007) 26(30):5512-28. doi: 10.1002/sim.3148

28. Eisenhauer EA, Therasse P, Bogaerts J, Schwartz LH, Sargent D, Ford R, et al. New Response Evaluation Criteria in Solid Tumours: Revised RECIST Guideline (Version 1.1). Eur J Cancer (2009) 45(2):228-47. doi: 10.1016/ j.ejca.2008.10.026

29. Waisberg DR, Pinheiro RS, Nacif LS, Rocha-Santos V, Martino RB, Arantes RM, et al. Resection for Intrahepatic Cholangiocellular Cancer: New Advances. Transl Gastroenterol Hepatol (2018) 3:9. doi: 10.21037/tgh.2018.08.03

30. Cho JY, Han HS, Yoon YS, Hwang DW, Jung K, Kim JH, et al. Preoperative Cholangitis and Metastatic Lymph Node Have a Negative Impact on Survival After Resection of Extrahepatic Bile Duct Cancer. World J Surg (2012) 36 (8):1842-7. doi: 10.1007/s00268-012-1594-0

31. Hong H, Jiang L, Lin Y, He C, Zhu G, Du Q, et al. TNF-Alpha Promotes Lymphangiogenesis and Lymphatic Metastasis of Gallbladder Cancer Through the ERK1/2/AP-1/VEGF-D Pathway. BMC Cancer (2016) 16:240. doi: 10.1186/s12885-016-2259-4

32. Jeong WK, Jamshidi N, Felker ER, Raman SS, Lu DS. Radiomics and Radiogenomics of Primary Liver Cancers. Clin Mol Hepatol (2019) 25 (1):21-9. doi: 10.3350/cmh.2018.1007

33. Xia W, Chen Y, Zhang R, Yan Z, Zhou X, Zhang B, et al. Radiogenomics of Hepatocellular Carcinoma: Multiregion Analysis-Based Identification of Prognostic Imaging Biomarkers by Integrating Gene Data-a Preliminary Study. Phys Med Biol (2018) 63(3):035044. doi: 10.1088/1361-6560/aaa609

Conflict of Interest: The authors declare that the research was conducted in the absence of any commercial or financial relationships that could be construed as a potential conflict of interest.

Publisher's Note: All claims expressed in this article are solely those of the authors and do not necessarily represent those of their affiliated organizations, or those of the publisher, the editors and the reviewers. Any product that may be evaluated in this article, or claim that may be made by its manufacturer, is not guaranteed or endorsed by the publisher.

Copyright (C) 2021 Liu, Liang, Ruan and Yan. This is an open-access article distributed under the terms of the Creative Commons Attribution License (CC BY). The use, distribution or reproduction in other forums is permitted, provided the original author(s) and the copyright owner(s) are credited and that the original publication in this journal is cited, in accordance with accepted academic practice. No use, distribution or reproduction is permitted which does not comply with these terms. 